\title{
DISTRIBUTION OF CONTRACTIBLE EDGES AND THE STRUCTURE OF NONCONTRACTIBLE EDGES HAVING ENDVERTICES WITH LARGE DEGREE IN A 4-CONNECTED GRAPH
}

\author{
Shunsuke NaKamura \\ Department of Mathematics \\ Tokyo University of Science \\ Kagurazaka 1-3 \\ Shinjuku-Ku, Tokyo 162-8601, Japan \\ e-mail: nakamura_shun@rs.tus.ac.jp
}

\begin{abstract}
Let $G$ be a 4 -connected graph $G$, and let $E_{c}(G)$ denote the set of 4 contractible edges of $G$. We prove results concerning the distribution of edges in $E_{c}(G)$. Roughly speaking, we show that there exists a set $\mathcal{K}_{0}$ and a mapping $\varphi: \mathcal{K}_{0} \rightarrow E_{c}(G)$ such that $\left|\varphi^{-1}(e)\right| \leq 4$ for each $e \in E_{c}(G)$.
\end{abstract}

Keywords: 4-connected graph, contractible edge, cross free.

2010 Mathematics Subject Classification: 05C40.

\section{REFERENCES}

[1] K. Ando and Y. Egawa, Edges not contained in triangles and the distribution of contractible edges in a 4-connected graph, Discrete Math. 308 (2008) 3449-3460. https://doi.org/10.1016/j.disc.2007.07.013

[2] K. Ando, Y. Egawa, K. Kawarabayashi and M. Kriesell, On the number of 4contractible edges in 4-connected graphs, J. Combin. Theory Ser. B 99 (2009) 97-109. https://doi.org/10.1016/j.jctb.2008.04.003

[3] R. Diestel, Graph Theory, 5th Edition (Springer-Verlag, Heidelberg, 2017).

[4] K. Kotani and S. Nakamura, The existence condition of a 4-connected graph with specified configurations, Far East J. Appl. Math. 98 (2018) 51-71.

https://doi.org/10.17654/AM098010051

Received 20 March 2018

Revised 4 February 2019

Accepted 6 May 2019 